\title{
La fabrication du travail non qualifié
}

Analyser les obstacles à la valorisation

\section{Didier Demazière et Emmanuelle Marchal}

\section{(2) OpenEdition}

\section{Journals}

Édition électronique

URL : http://journals.openedition.org/travailemploi/8583

DOI : $10.4000 /$ travailemploi.8583

ISSN : 1775-416X

Éditeur

DARES - Ministère du Travail

Édition imprimée

Date de publication : 1 juillet 2018

Pagination : 5-30

ISSN : 0224-4365

Référence électronique

Didier Demazière et Emmanuelle Marchal, «La fabrication du travail non qualifié », Travail et Emploi [En ligne], 155-156 | Juillet-décembre 2018, mis en ligne le 11 juillet 2019, consulté le 24 septembre 2020 URL : http://journals.openedition.org/travailemploi/8583; DOI : https://doi.org/10.4000/travailemploi. 8583 


\title{
Introduction
}

\section{La fabrication du travail non qualifié}

\author{
Analyser les obstacles à la valorisation
}

\author{
Didier Demazière ${ }^{*}$ Emmanuelle Marchal ${ }^{*}$
}

Wen qualifié, travail non qualifié, salariés non qualifiés, main-d'œuvre non qualifiée, longue des usages de cette expression, qui circule dans l'espace public où elle semble frappée du sceau de l'évidence. Elle alimente nombre de discours portant sur le travail contemporain : annonces de la montée en gamme des compétences et de la disparition du travail non qualifié (les nouvelles technologies le balaieraient à terme), identification de celui-ci comme source d'un chômage persistant (les chômeurs compteraient trop de non-qualifiés), effritement des qualifications avec le développement de la polyvalence (nombre de métiers seraient frappés de déqualification), etc. La diffusion de cette thématique de la non-qualification justifie que l'on fasse retour sur cette question (Méda, Vennat, 2004 ; Rose, 2012).

La perspective privilégiée dans ce dossier est tracée autour d'une question centrale : par quels processus le travail est-il non qualifié, comment certaines activités et certains travailleurs sont-ils considérés comme non-qualifiés ou traités comme tels ? Ces questions ne reçoivent pas de réponses générales ou invariantes, car le phénomène de la «non-qualification » a des limites différentes d'un pays à l'autre, évolue avec le temps, se déplace entre les secteurs d'activité, touche des populations diverses. Ces variations indiquent que le travail non qualifié ne peut être rabattu sur un ensemble de traits intrinsèques ou de propriétés stables. Les impasses de l'approche substantielle sont d'ailleurs bien connues. Un argument majeur, reposant sur la critique de l'équation "non-qualification = absence de qualités », a été déployé dans plusieurs directions : un travail qui n'exigerait aucune qualité n'aurait aucune utilité et donc n'existerait pas, de sorte que toutes les formes de travail impliquent la mobilisation de qualités ; tout travailleur est dépositaire de qualités accumulées à travers sa formation et ses expériences qu'il actualise dans le cours de son activité ; tout processus de recrutement

\footnotetext{
* Centre de sociologie des organisations (CNRS/Sciences Po); didier.demaziere@ sciencespo.fr.

** Centre de sociologie des organisations (CNRS/Sciences Po); emmanuelle.marchal@ sciencespo.fr.
} 
compte des opérations de sélection qui sont adossées à des procédures et critères visant à repérer des qualités chez les candidats, etc. Et pourtant l'expression «non qualifié » est une catégorie pertinente. Pertinente quand on la considère comme le résultat de processus d'attribution, dont nous proposons l'exploration.

Car il ne s'agit pas seulement de soutenir que tout poste de travail et tout travailleur mettent en jeu un nombre minimal de qualités ou de compétences, mais plutôt de considérer le travail non qualifié comme la résultante d'un déficit de leur reconnaissance et de leur valorisation. Il incorpore un «voir comme», un « compter pour» : ce qui est non qualifié, c'est ce qui est vu comme tel, ce qui est compté pour tel. Cette posture théorique, déjà expérimentée ailleurs (JANY-CATRICE, 2007 ; CHAUvIN, 2010), n'est pas relativiste pour autant. Car si l'expression «non qualifié » est désormais admise et retenue comme pertinente pour rendre compte d'évolutions contemporaines du travail, c'est parce qu' elle est adossée à des conventions sociales ; des conventions de perception, de mesure, de valorisation, qui sont suffisamment cristallisées et stabilisées pour qu'elles fassent sens, circulent et organisent des rapports sociaux de travail. Ces conventions, plus ou moins institutionnalisées, ne couvrent pas de manière uniforme le spectre des emplois et des postes. Ainsi, dans nombre de secteurs industriels, des conventions collectives ont fixé des classifications professionnelles ouvrières. Et celles-ci délimitent la frontière entre des emplois d'ouvrier professionnel ou qualifié, correspondant à des métiers dont l'exercice exige une formation et un apprentissage, et des emplois d'ouvrier spécialisé et de manœuvre ne nécessitant aucune formation ou, au plus, une adaptation rudimentaire. Mais ces catégories n'ont pas d'équivalent dans l'archipel des employés, où la différenciation entre qualifié et non qualifié est plus flottante, alors même que s'y développe le travail non qualifié ${ }^{1}$. De manière convergente, la nomenclature des professions et catégories socioprofessionnelles distingue de longue date les ouvriers « qualifiés » et « non qualifiés » (à l'échelle des 24 postes) (DESROSIÈRES, THÉVENOT, 1988) mais n'introduit pas explicitement ce critère pour les employés, même si des définitions des employés non qualifiés sont proposées (AMOsSÉ, Chardon, 2006).

Toutefois, dans une visée opératoire pour notre perspective d'analyse, nous pouvons appréhender la non-qualification comme un phénomène de faible valorisation du travail, caractérisé par l'occupation de positions basses dans les hiérarchies des emplois et des rémunérations. De fait, elle est souvent combinée, du moins dans le contexte actuel de croissance de la flexibilité et de fragilisation de la relation d'emploi, à une distance à la norme de l'emploi stable. La minoration statutaire contribue à la non-qualification et la renforce, dès lors que la qualification du travail n'est pas réduite à l'inscription

1. Ce constat est lui-même dépendant de conventions de mesure. Les débats sur la mesure se sont concentrés sur la catégorie des employés, plusieurs conventions ayant été proposées. L'une met l'accent sur le niveau de salaire et la durée de la formation (BURNOD, CHENU, 2001), ce qui conduit à associer la non-qualification à des propriétés caractérisant les salariés, en l'espèce ceux qui sont « peu diplômés et peu payés ». Une autre retient le critère d'adéquation entre le contenu de la profession et les spécialités de formation des actifs qui exercent ce métier (CHARDON, 2001 ; AMOSSÉ, Chardon, 2006). 
dans des classifications professionnelles mais s'entend plus largement comme la configuration d'une place dans les systèmes d'emploi. Les modes de (dé)valorisation et de (non-)reconnaissance de cette place sont multiples et peuvent être déclinés en termes de protection statutaire, d'accès à des droits sociaux, de pénibilité du travail, de niveau de rémunération, de flexibilité temporelle, d'intensité des cadences et de contraintes de toutes sortes.

De tels constats nous incitent à sortir du cadre des organisations ou des secteurs d'activité où les classifications professionnelles sont codifiées et fixent des limites stabilisées entre ce qui est alors désigné comme les emplois qualifiés et les emplois non qualifiés. Privilégiant une perspective dynamique, nous nous intéressons à ce qui peut porter atteinte à la reconnaissance des qualifications, y compris lorsque l'on est au chômage, simple candidat à l'emploi, intérimaire ou employé. Une telle perspective permet de rendre compte de la multiplicité des parties prenantes engagées dans les processus de (non-)reconnaissance : salariés, employeurs, recruteurs, collègues, syndicats, entreprises, intermédiaires publics ou privés, etc. Elle permet également de pointer la malléabilité et la fragilité des qualifications, qui sont questionnées, mises en cause ou négociées, en de nombreuses occasions.

Qu'est-ce qui contribue à fragiliser la reconnaissance des qualifications, à freiner les mécanismes de conversion des qualités en valeurs ? Sans prétendre dresser un panorama complet de tels freins, le choix des textes réunis dans ce dossier vise à examiner ce qui contribue à faire échouer les processus de reconnaissance de la valeur du travail dans des activités variées : l'aide à domicile pour laquelle François-Xavier Devetter et Emmanuelle Puissant dégagent un faisceau de mécanismes économiques contribuant à maintenir les emplois dans les catégories non qualifiées ; l'hôtellerierestauration où Sylvie Monchatre montre comment sont investies les opérations de recrutement destinées à pourvoir des postes non qualifiés ; le commerce où Pauline Grimaud étudie comment l'extension du travail dominical contribue à redéfinir les qualifications des employés ; la logistique où Lucas Tranchant examine comment le recours à l'intérim de masse est un vecteur de «disqualification professionnelle » et, dans une perspective non sectorielle, le rôle de Pôle emploi dans la définition des qualifications des demandeurs d'emploi que met en évidence Hadrien Clouet. De fait, la fabrication de la non-qualification se nourrit de nombreux éléments : profusion d'offres de travail, réduction du périmètre des conventions collectives, faiblesse des organisations syndicales, fragilité de la professionnalisation d'activités émergentes, abondance d'une caractéristique productive, reconfiguration des organisations du travail, forte rotation de la main-d'œuvre, etc.

Dans les développements qui suivent, nous nous appuyons sur les textes du dossier pour prêter attention aux enjeux qui prennent place en amont, sur le marché du travail, puis aux portes des entreprises et enfin à l'intérieur de celles-ci. Nous dégageons ainsi différents processus de fabrication du travail non qualifié en soulignant, en trois temps, comment sont fragilisés : des demandeurs d'emploi face aux opportunités d'emploi ; des candidats face aux recruteurs ; des travailleurs précaires face aux employeurs. 


\section{Le terreau du développement d'un marché du travail non qualifié}

L'abondance ou la rareté de la main-d'œuvre disponible et mobilisable pour effectuer une activité donnée sont des facteurs contribuant à la (dé)qualification de celle-ci. La sociologie des professions a amplement montré comment des groupes professionnels cherchent à contrôler leur marché du travail en établissant des barrières à l'entrée qui remplissent deux fonctions principales : s'assurer que les prétendants ont suivi un cursus de formation les préparant à la pratique professionnelle et contingenter les effectifs des nouveaux entrants de manière à préserver une rareté relative (АВвоTт, 1988). En ce sens, la maîtrise de la démographie professionnelle - entendue comme le contrôle du nombre de travailleurs pouvant prétendre exercer une activité - est un levier majeur de la qualification. Elle permet d'accroître leur légitimité sociale, de renforcer leur reconnaissance, et d'améliorer leurs revenus (DEMAZIÈRE, GADÉA, 2009). À l'inverse, l'absence d'une telle maitrise, et plus encore la fabrication d'une maind'œuvre potentielle abondante, sont des conditions favorables à une non-qualification.

De telles conditions sont réunies dans les activités de services à domicile, comme nous allons le voir. La croyance en l'existence de potentialités d'emplois dans telle ou telle activité se décline à son tour dans le travail quotidien des agents de Pôle emploi, qui orientent une partie des demandeurs d'emploi vers les gisements repérés. Elle est relayée également par les employeurs qui créent des conditions propices à une mise en concurrence des candidats, en engrangeant des candidatures de manière excédentaire pour faire face à des demandes souvent imprévisibles, partielles ou saisonnières. Nous verrons ensuite que les conditions de la rencontre entre les offres et les demandes sont fortement affectées par ce mode de fonctionnement. Marquées par des asymétries d'information, elles ne permettent pas de prendre réellement en compte les besoins de la main-d'œuvre dont la position se trouve fragilisée.

\section{La constitution de réserves de main-d'œuvre}

Les services d'aide à domicile, au cœur du texte de F.-X. Devetter et E. Puissant, fournissent un exemple éclairant de la manière dont se constituent des réserves de main-d'œuvre. Dans ces services réputés en tension, où les employeurs connaîtraient des difficultés permanentes de recrutement, des mesures sont prises pour cibler différentes populations en vue d'alimenter des viviers de candidatures. L'objectif est de s'adresser à des catégories de main-d'œuvre peu mobilisées afin de les mettre au travail, en particulier des mères de famille et des femmes immigrées susceptibles de prendre ou de reprendre une activité abandonnée pour élever leurs enfants. La main-d'œuvre immigrée est directement pressentie pour cette activité, avec l'idée qu'elle pourra y exprimer ses compétences « naturelles » pour s'occuper des personnes âgées. Les arguments visant à attirer des postulants sont aussi traduits en actions de communication, comme lorsque les entreprises du secteur font témoigner des salariés qui vantent la 
possibilité de concilier un tel travail avec leurs contraintes familiales. Ces objectifs visant à mobiliser une main-d'œuvre latente, pour des emplois à horaires discontinus, sont relayés par des politiques publiques soutenant le développement des services à la personne : via le revenu de solidarité active (RSA) qui permet les cumuls d'aides et de revenus d'activités à temps partiel ${ }^{2}$, via le financement de permis de conduire ou via les exonérations de charges sociales et fiscales consenties aux employeurs.

Les services à la personne présentent de fortes spécificités, en particulier parce qu'ils ont été constitués en instrument des politiques sociales et des politiques d'emploi (DevetTer et al., 2009). Mais la formation de viviers de main-d'œuvre ne se limite pas à cette activité. Elle se retrouve à chaque fois qu'interviennent des prestataires de services et dans les secteurs où le turnover et les besoins sont élevés. Elle peut prendre appui sur la mobilisation de réseaux ethniques comme cela a été observé dans le bâtiment (Jounin, 2004) ou le maraîchage (DÉCOSSE, 2012), sur l'appel à l'État en vue de pouvoir recruter des immigrés sans-papiers dans l'hôtellerie et la restauration (BARRON et al., 2011), et, il y a plus longtemps, sur l'importation de main-d'œuvre immigrée depuis les territoires coloniaux (MORICE, РОTOT, 2010). La capacité des entreprises à produire l'abondance de main-d'œuvre est le signe d'un déséquilibre marqué des rapports sociaux de production, son pendant étant l'incapacité de la main-d'œuvre ainsi visée à mobiliser des ressources collectives pour contrecarrer ces stratégies. Le contexte global, marqué en France par un chômage important et durable, constitue à cet égard un facteur favorable à la non-qualification, à laquelle l'armée de réserve (MARX, 1867) est particulièrement exposée. Poussant plus loin encore le raisonnement, on peut se demander si l'annonce périodique de «pénuries de main-d'œuvre », notamment dans le secteur de la restauration, ne correspond pas à une stratégie patronale destinée à entretenir l'appel de la main-d'œuvre dans ce secteur. Le fait est que c'est là où le turnover est le plus élevé et où les conditions de travail sont marquées par de fortes fluctuations que de telles pénuries sont soulignées par les employeurs et leurs représentants, qui se plaignent de difficultés de recrutement (ZunE, 2014).

Dans ce contexte, les intermédiaires de l'emploi contribuent à orienter une partie des demandeurs d'emploi vers ces supposés gisements d'emplois, abondant ainsi les réservoirs de main-d'œuvre. Sur ce point, le texte d'H. Clouet apporte de nombreux éléments, à partir de l'observation ethnographique des interactions entre conseillers à l'emploi et demandeurs d'emploi. Il montre comment les entretiens d'inscription et de suivi sont des moments de discussion et de réglage des qualités des demandeurs d'emploi et, partant, des aspirations et prétentions qu'ils peuvent légitimement nourrir. Les caractéristiques de leurs profils apparaissent malléables, ajustées dans l'échange et révisées dans l'enregistrement informatique. Cela permet alors de configurer les offres d'emploi pouvant correspondre aux propriétés répertoriées des demandeurs,

2. Des travaux montrent néanmoins que « l'attirance » des employeurs pour les bénéficiaires du RSA est très limitée. Les employeurs accordent la priorité à la motivation des candidats qui doivent supporter des conditions de travail difficiles et ne se tournent pas volontiers vers ce type de main-d'œuvre jugée inemployable (BÉRAUD et al., 2015). 
à ce qu'ils recherchent, à ce qu'ils peuvent prétendre, à ce qu'ils sont disposés à accepter. Un enjeu de ces entretiens est de rapprocher demandes et offres, ce qui passe souvent par une redéfinition des qualités des demandeurs en vue de les ajuster aux opportunités disponibles ${ }^{3}$. H. Clouet analyse finement tout ce qui peut amener les demandeurs d'emploi à accepter des propositions d'emploi de moindre qualité que ce qu'ils envisageaient. Entre les formatages inhérents au logiciel et aux algorithmes d'appariement, les pressions que subissent les conseillers, leur conviction d'avoir affaire à des demandes difficiles à satisfaire, la tendance à réévaluer et à dévaloriser parfois les parcours entachés de périodes de chômage, de nombreux facteurs conduisent à opérer des réajustements dans un sens ou dans un autre. Ces analyses montrent ainsi comment les conseilleurs contribuent à la régulation des qualifications.

Les mobilités entre emplois, qui passent par le chômage, sont moins des occasions de promotion que le signal de la fragilité de la position du demandeur d'emploi. Des travaux portant sur des carrières affectées par le chômage montrent que la plupart du temps, les changements de métiers ne sont pas des choix délibérés, mais résultent de l'urgence de la situation et de l'obligation de travailler. Le chômeur accepte de changer parce qu'il ne peut faire autrement. Or, les ouvriers et les employés non qualifiés sont particulièrement concernés par ces mobilités professionnelles contraintes (MATUS, Prokovas, 2014 ; Flamand, 2016), comme si l'expérience acquise dans un type de métier ne pouvait être valorisée dans un autre emploi du même type. Cependant, toutes les personnes qui occupent à un moment ou à un autre des emplois non qualifiés ne sont pas logées à la même enseigne. Le niveau de diplôme opère un partage parmi les jeunes ${ }^{4}$, entre ceux pour qui les emplois non qualifiés représentent des portes d'entrée sur des marchés internes et ceux dont le niveau de formation est trop faible pour envisager un parcours ascendant (Amossé et al., 2011). De ce point de vue, les activités d'aide à domicile, d'employés de maison ou de nettoyage, sont considérées comme des « activités enfermantes », dont les personnes sortent très difficilement (ESTRADE, 2008, p. 35).

\section{Des conditions de rencontre marquées par de fortes asymétries}

Dans ces situations, les conditions de la rencontre entre la main-d'œuvre et les employeurs ne laissent pas de place à des processus de reconnaissance et génèrent de fortes asymétries. L'enquête Offre d'emploi et recrutement (Ofer, 2016 ; encadré) de la Direction de l'animation de la recherche, des études et des statistiques (Dares) nous permet d'identifier quels canaux de recrutement sont activés, quels éléments sont

3. Ces constats rejoignent d'autres travaux montrant la malléabilité des qualifications de la main-d'œuvre. Car celles-ci se prêtent en effet à de nombreuses définitions comme l'illustrent les multiples conseils diffusés sur internet, où l'on incite les demandeurs d'emploi à se présenter en des termes correspondant aux offres d'emploi pour être repérés par les moteurs de recherche (MARCHAL, 2015).

4. Les jeunes sont particulièrement concernés par l'instabilité des contrats sur des emplois non qualifiés, avec des taux de transition élevés vers le chômage, vers les temps partiels et vers les contrats courts. Mais la situation des seniors s'est récemment dégradée, et ils sont de plus en plus touchés par ces phénomènes d'instabilité (PICART, 2014). 
demandés aux candidats par les employeurs et quelles sont les méthodes de sélection utilisées. Ce cadrage statistique donne une image de la façon dont on recrute les candidats pour des emplois peu qualifiés, que les analyses développées dans les textes de ce dossier viennent détailler et enrichir.

\section{ENCADRÉ}

\section{L'enquête Offre d'emploi et recrutement 2016}

L'enquête Offre d'emploi et recrutement (Ofer) a été menée en 2016 par la Dares auprès de 8510 établissements ayant recruté entre septembre et décembre 2015. L'interrogation porte sur leur dernier nouveau recrutement, effectué en contrat à durée indéterminée (CDI) ou en contrat à durée déterminée (CDD) de plus d'un mois entre le $1^{\text {er }}$ septembre et le 30 novembre 2015. Un recrutement est considéré comme «nouveau » dès lors que la personne recrutée a quitté l'établissement depuis au moins six mois et n'a pas été embauchée dans le même établissement entre septembre 2013 et août 2015. Le champ de l'enquête couvre l'ensemble des établissements d'au moins un salarié du secteur concurrentiel non agricole. Le détail de la méthodologie adoptée et les principaux résultats de cette enquête sont présentés dans la première publication de la Dares (BERGEAT, RÉMY, 2017). Deux autres publications ont suivi, l'une portant sur les recrutements en CDD (RÉmY, 2017) et l'autre sur la mobilisation d'internet (BERGEAT et al., 2018).

Pour cette introduction, nous avons centré l'exploration statistique sur les 3396 opérations de recrutement (soir près de $40 \%$ du total des interrogations d'Ofer 2016) qui ont concerné des ouvriers et des employés non qualifiés. Nous avons également isolé cinq sous-populations qui correspondent (ou sont proches des populations) à celles dont il est question dans les articles de ce dossier, mais ne les recouvrent pas complètement :

- les employés du commerce $(\mathbf{N}=\mathbf{7 3 0})$ : vendeurs alimentaires, magasiniers, employés de libre-service, caissiers, télévendeurs ;

- les employés de l'hôtellerie et de la restauration $(\mathrm{N}=618)$ : serveurs, commis de salle, barmen, équipiers, aides de cuisine, employés d'hôtellerie ;

- les employés des services à domicile $(\mathbf{N}=\mathbf{2 7 6})$ : aides à domicile, assistantes maternelles, gardiennes d'enfants, employés de maison, gardiens ;

- les nettoyeurs $(\mathbf{N}=\mathbf{3 0 2})$ : agents de propreté, de service, d'entretien, de ménage, de nettoyage de bureaux, locaux ;

- les ouvriers du bâtiment $(\mathbf{N}=\mathbf{5 0 1})$ : manœuvres ou aides-couvreurs (gros œuvre), aides-électriciens, menuisiers ou peintres (petit œuvre).

Nous avons mené des régressions pour évaluer la probabilité d'opérer un recrutement 1) pour un emploi non qualifié ( $\mathrm{N}=3$ 396) plutôt que pour un autre emploi et 2) pour un emploi appartenant à chacune des cinq sous-populations d'emplois non qualifiés, plutôt que pour un autre emploi non qualifié. Nous présentons dans cette introduction certains des résultats significatifs « toutes choses égales par ailleurs ». 
L'enquête Ofer $2016^{5}$ permet tout d'abord de souligner les caractéristiques des modes de recherche de la main-d'œuvre non qualifiée dans le domaine des services à domicile. On sait que les gestionnaires de services privés, publics ou associatifs multiplient les initiatives en prospectant tous azimuts des candidats. On voit dans $O f e r$ qu'ils mobilisent la plupart des modes de sourcing cités dans l'enquête - et davantage que les employeurs qui pourvoient d'autres emplois non qualifiés -, les candidatures spontanées, les salons de l'emploi, les candidatures reçues à l'occasion de recrutements précédents et les annonces sur internet ${ }^{6}$. Cette sur-sollicitation permet d'entretenir des viviers de candidatures pour faire face à des demandes imprévues d'aide à domicile et de garder une marge de manœuvre pour opérer une sélection au sein de ces viviers. C'est donc une caractéristique du recrutement dans ce secteur où la concurrence est entretenue par les pratiques des recruteurs.

$\mathrm{La}$ «production » d'une telle concurrence s'observe également dans des secteurs qui recourent à l'intérim ${ }^{7}$, les agences de travail temporaire constituant d'abondants fichiers de candidatures. Si l'on se place du point de vue du postulant, l'obtention d'une inscription dans une agence ne garantit pas celle d'un emploi et se double d'une attente de mission pour laquelle les inscrits se trouvent en compétition. Ce qui a été décrit à propos de journaliers de Chicago (CHAUvin, 2010) se retrouve pour l'accès aux entrepôts de logistique analysé par $\mathbf{L}$. Tranchant. Les candidats à l'intérim sont maintenus dans l'attente de missions qui leur sont souvent vitales. Ils sont soumis au pouvoir de convocation des agences et ne peuvent s'éloigner de ces dernières sans risquer de laisser passer des opportunités. Il s'agit non seulement de se manifester auprès des agences pour réaffirmer en permanence sa disponibilité, mais aussi de répondre positivement lorsqu' une mission est proposée. Même si celle-ci ne dure que quelques heures, il vaut mieux l'accepter pour ne pas être considéré comme un «mauvais » candidat et risquer de ne plus recevoir de propositions. Les convocations par SMS renforcent l'impression d'être en compétition et l'exigence de disponibilité immédiate, d'autant qu'il s'agit souvent de textos collectifs et impersonnels (RosinI, 2012).

Ce que l'on observe pour les intérimaires, à savoir la nécessité d'accepter des missions dont on ne connaît ni le lieu, ni la durée, ni le contenu, est courant dans l'accès à des emplois de faible niveau de qualification. Comme nous venons de le voir, les candidats ne choisissent pas réellement les emplois pour lesquels ils postulent lorsqu'interviennent des agences d'emploi ou des prestataires de services. L'information est capturée par les intermédiaires qui affectent la main-d'œuvre là où se trouvent les besoins. Même lorsque des annonces sont publiées, comme c'est le cas

\footnotetext{
5. Nous tenons à remercier tout particulièrement Marie-Sophie Dumont, ingénieur en production et méthodologie d'enquête au Centre de sociologie des organisations, qui a opéré tous les traitements statistiques que nous présentons ici. 6. Ces résultats sont issus d'une régression qui tient compte des principales caractéristiques des entreprises qui recrutent et des conditions dans lesquelles s'effectue le recrutement (durée du processus de recrutement, nombre de personnes investies, diffusion, etc.).

7. L'enquête $O f e r$ (2016) ne permet pas de faire apparaître le rôle de l'intérim, seuls les recrutements dans les emplois salariés étant pris en compte.
} 
dans le domaine des services à domicile ${ }^{8}$, il s'agit plutôt d'appels à des candidatures spontanées, d'offres génériques ne comportant qu'un intitulé d'emploi, sans descriptif des conditions d'emploi et de travail proposées. De ce fait, la plupart des candidats aux emplois non qualifiés postulent « en aveugle », dans les lieux où ils sont sélectionnés, sans choisir l'emploi qui correspond le mieux à leurs attentes en termes de distance au domicile ou de répartition du temps de travail. De telles pratiques ne peuvent qu'alimenter le turnover sur ces emplois.

Selon les données de l'enquête $O f e r$ 2016, le moyen le plus fréquent pour pourvoir des emplois non qualifiés est la candidature spontanée (graphique 1). C'est là une caractéristique que l'on ne retrouve pas dans le recrutement pour des emplois qualifiés, de professions intermédiaires ou de cadres (BERGEAT, RÉmY, 2017). Il revient donc aux postulants aux emplois de faible qualification de pressentir l'employeur, de tenter leur chance, sans avoir l'assurance qu'existe une opportunité d'emploi qui soit dans leurs cordes. De fortes disparités d'un type d'emploi à l'autre apparaissent toutefois, de sorte que trois modes de recrutement peuvent être distingués dans le domaine du travail non qualifié.

GRAPHIQUE 1 - Les canaux effectifs de recrutement dans les cinq types d'emplois non qualifiés

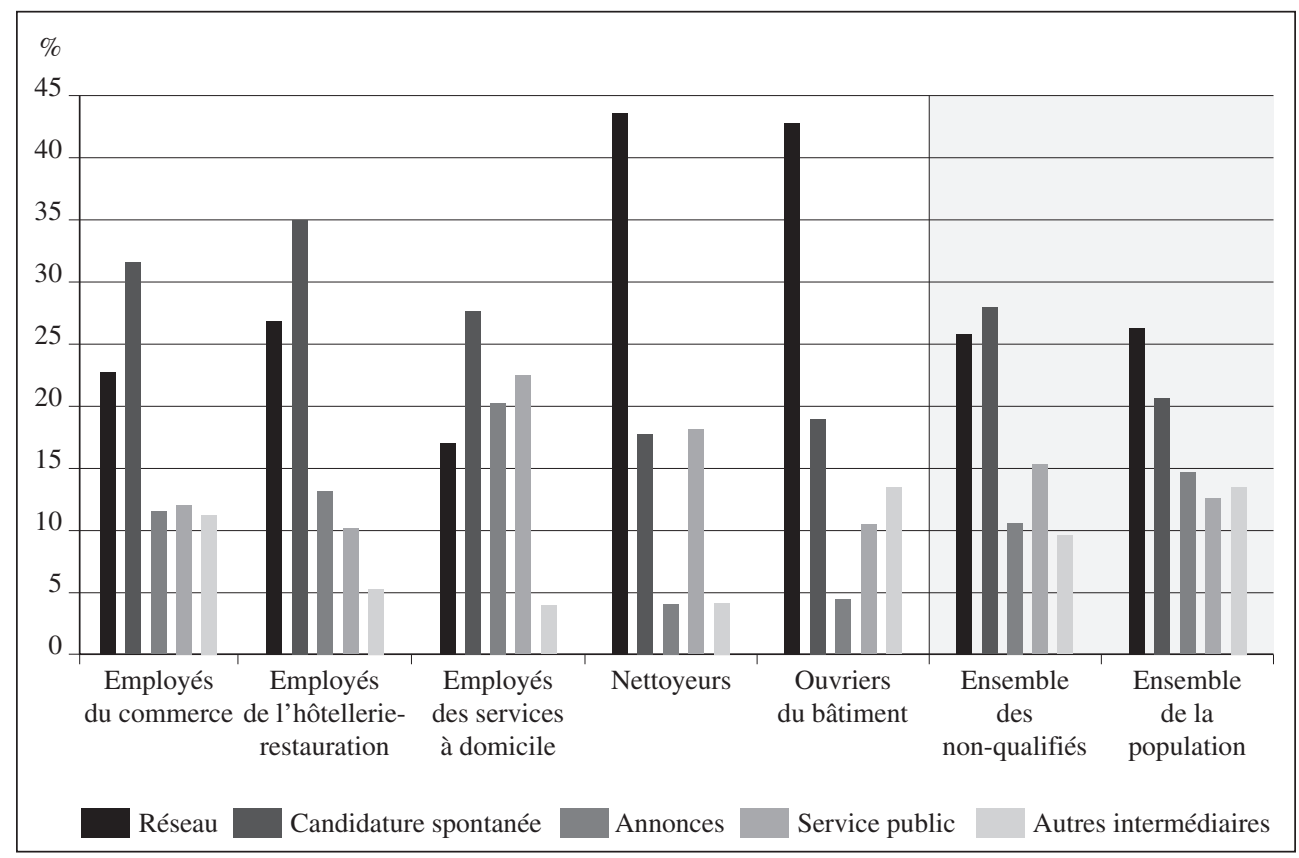

Champ : ensemble des nouveaux recrutements en CDI ou CDD de plus d'un mois dans les établissements d'au moins un salarié du secteur concurrentiel ; France entière. Données pondérées.

Source : Ofer, Dares, 2016.

8. Les annonces qui figurent dans les vitrines des agences d'emploi concernent plus souvent des emplois réclamant des qualifications précises. 
Le premier est typique des emplois du commerce et de l'hôtellerie-restauration, où les candidats se présentent en personne dans des boutiques, des cafés ou des hôtels pour déposer leur curriculum vitce (CV). Cette démarche est un signal de motivation pour l'employeur, qui y trouve aussi l'occasion d'évaluer la manière dont le candidat se présente ou s'exprime. Le même phénomène a été relevé dans la grande distribution (Rieucau, Salognon, 2013).

Dans le domaine des emplois à domicile, où des intermédiaires s'interposent entre candidats et employeurs, nous avons vu que ces derniers multiplient les démarches pour alimenter leurs viviers. De fait, on observe que pour ces emplois, les canaux qui vont permettre de recruter sont assez variés : les candidatures spontanées y jouent le premier rôle, comme ailleurs; le service public est le deuxième canal pourvoyeur de main-d'œuvre ; les recrutements par annonces sont très supérieurs à la moyenne ( $20,2 \%$ pour $14,6 \%$ lorsqu'est pris en compte l'ensemble de la population).

Le dernier mode de recrutement est caractéristique des emplois d'ouvriers non qualifiés du bâtiment et du nettoyage, pour lesquels les relations jouent un rôle déterminant. Plus de quatre emplois sur dix y sont pourvus par des réseaux (pour 26,2\% en moyenne), écrasant le rôle des autres canaux, et celui des annonces en particulier, qui s'avèrent très peu usités. Dans le détail, des intermédiaires plus spécialisés interviennent dans le recrutement des ouvriers du bâtiment, comme les écoles de formation où sont parfois directement recrutés les jeunes candidats en tant qu'apprentis.

L'urgence des recrutements est également un trait caractéristique des conditions d'embauche du personnel non qualifié. D'après Ofer 2016, cette urgence est particulièrement marquée pour les employés des services à domicile et pour les nettoyeurs : dans les deux cas, plus de quatre embauches sur dix doivent pouvoir être conclues en moins d'une semaine ${ }^{9}$. Il faut trouver une solution rapide pour une personne dépendante qui se réinstalle chez elle, ou remplacer au pied levé des employés ou des ouvriers qui ne se présentent pas à leur travail. On mesure ici l'importance des démissions ou des autres formes de renoncement à continuer de travailler dans les conditions offertes qui sont à la source des turnovers dans ces secteurs, mais aussi celle des arrêts de travail et des problèmes de santé ${ }^{10}$. Ailleurs, il s'agit de faire face à des besoins saisonniers, comme dans les emplois de l'hôtellerie-restauration où trois recrutements sur dix se font également en urgence.

De tels délais ne sont pas propices à négocier quoi que ce soit au moment de l'embauche. Les besoins spécifiques des candidats en particulier, peuvent difficilement être pris en compte. Un dernier indice de leur faible capacité de négociation concerne les prétentions salariales. La demande de prétention ou de précision sur le salaire actuel de la part d'une entreprise qui recrute vise à tester si elle pourra satisfaire la demande du

9. Voir note 6.

10. Le nombre d'accidents du travail dans la branche des « activités de santé, nettoyage et travail temporaire » où sont classés les services à domicile est particulièrement élevé. Il a augmenté globalement de 17,7 \% entre 2001 et 2015 , mais de $81 \%$ pour les femmes tandis qu'il a baissé de $28,4 \%$ pour les hommes. Sur la même période et dans le même groupe d'activités, le nombre d'accidents de trajets a augmenté de 43,3\% pour les femmes (CHAPPERT, THERRY, 2017). 
candidat, ou à apprécier si, compte tenu de la valeur de ce dernier, elle aurait avantage à le rejeter ou à s'aligner sur les prétentions en question. Cette demande suppose donc l'existence d'une marge de négociation d'un côté ou de l'autre. D'après l'enquête Ofer 2016, la demande de prétentions salariales est significativement moins fréquente lorsqu'il s'agit de recruter pour un emploi non qualifié que pour un autre emploi. On observe par ailleurs que cette demande est particulièrement faible dans l'hôtellerierestauration et pour embaucher des nettoyeurs ( $24 \%$ ), tandis qu'elle remonte pour les ouvriers non qualifiés du bâtiment, probablement parce qu'ils peuvent bénéficier de primes ou d'indemnités ajustées à chaque situation.

\section{Une sélection focalisée sur des critères étrangers à la qualification}

Que les recrutements s'effectuent en urgence n'empêche pas de rejeter des candidatures et d'opérer une sélection drastique de la main-d'œuvre. Cela conduit à s'intéresser aux qualités recherchées par les employeurs et à la manière dont ils s'y prennent pour les repérer à l'occasion des recrutements. Il a déjà été abondamment souligné que tous les postes de travail, y compris ceux qui correspondent aux tâches apparemment les plus simples, requièrent des savoirs techniques et des compétences implicites (Rose, 2012). C'est donc l'invisibilité des compétences, et non leur absence, qui nourrit la désignation du travail non qualifié ${ }^{11}$.

Deux explications sont généralement avancées pour rendre compte de la manière dont cette dévalorisation des compétences est légitimée dans les discours. La première insiste sur la naturalisation et la banalisation de compétences qui sont renvoyées à des savoir-être et à des propriétés extraprofessionnelles. Cette naturalisation est typique de métiers fortement féminisés (GUICHARD-Claudic et al., 2008), des activités de care (CRESSON, GADREY, 2004), et plus généralement des activités dites relationnelles (JANY-CATRICE, 2007 ; Le FeUvRE et al., 2012). Elle conduit à assigner les femmes à des tâches reliées au travail domestique en se fondant sur la croyance qu'elles sont douées pour ces emplois (SCRINZI, 2013). De la même façon, les catégories ethniques sont mobilisées pour justifier l'affectation des hommes dans tels métiers du bâtiment (Jounin, 2004). La littérature féministe insiste en ce sens sur la manière dont les rapports de genre et les rapports racisés (KERGOAT, 2010 ; SCRINZI, 2013) sous-tendent l'attribution des emplois, la division du travail et l'absence de valorisation des tâches concernées. Ce peut être aussi une invisibilisation des compétences mobilisées dans le travail qui soutient la dévalorisation de celui-ci et des travailleurs ; une invisibilisation de compétences difficiles à énumérer, caractériser, formaliser (DussuET, 2016). Cela

11. Cette invisibilité des compétences peut se doubler d'une invisibilisation du travail et des personnes. C'est le cas par exemple pour les nettoyeurs, dont la présence est considérée comme embarrassante dans les crèches, les hôpitaux ou les bureaux où ils travaillent. Mais elle n'empêche pas le contrôle des tâches que les travailleurs des entreprises clientes s'arrogent d'autant plus facilement le droit d'évaluer qu'elles sont assimilées à des tâches domestiques (REYSSAT, 2016). 
est avancé à propos des services à domicile qui ont été historiquement externalisés de la sphère domestique vers la sphère publique et dont les emplois sont essentiellement occupés par des femmes. N'étant pas objectivables, les compétences ne pourraient être enseignées à l'école pour être sanctionnées par des diplômes, ni faire l'objet d'apprentissages explicites.

Au-delà de ces constats, il convient d'observer ce que la sélection de la maind'œuvre non qualifiée valorise, néglige ou écarte. La littérature insiste en effet sur le caractère « informel » des recrutements qui serait une pratique répandue pour ce type de main-d'œuvre. Mais une lecture transversale des articles qui composent ce dossier et les résultats de l'enquête Ofer 2016 permettent de dépasser cette idée, en repérant les mécanismes à l'œuvre dans la sélection. En effet, la sélection ne disparaît pas quand il s'agit de mobiliser du travail ou des travailleurs non qualifiés, mais se déplace vers des qualités qui sont sources de différenciations non qualifiantes. Trois dimensions de cette sélection sont explorées : l'invalidation des qualifications certifiées et, en particulier, des diplômes ; la diversité des exigences formulées à l'égard des candidats ; les conditions dans lesquelles la sélection se déroule.

\section{Minoration des compétences certifiées et des diplômes}

La détention de qualités certifiées par un titre scolaire ne garantit pas la reconnaissance d'une qualification ni même la simple validation de qualités valorisables dans les mondes du travail. En effet, les qualités acquises peuvent faire l'objet de négociation, ajustement, invalidation, comme le montre le texte d'H. Clouet à partir de l'analyse des interactions au guichet de Pôle emploi. Y sont produits des « jugements d'utilité » comme des « jugements d'inutilité » consistant à prendre en compte, ou non, des qualités qui peuvent pourtant être attestées, et à choisir de les enregistrer, ou non, dans l'application informatique qui renseigne les profils des demandeurs d'emploi. À un pôle, l'expérience accumulée peut être convertie par le conseiller en l'équivalent fonctionnel d'un titre. À l'autre pôle, la valeur d'un diplôme peut être discutée jusqu'à l'invalidation en fonction de l'appréciation de son ancienneté, de son lieu d'acquisition et du parcours professionnel postérieur à son obtention. Ce processus de déqualification, au sens d'effacement d'une qualité acquise, est légitimé par l'argument selon lequel la qualification peut faire obstacle à la sortie du chômage. L'objectif est de réviser à la baisse les qualités du demandeur, pour provoquer une hausse potentielle du nombre d'offres d'emploi lui correspondant. Ces négociations produisent des conditions favorables à la non-qualification ou à la minoration de la qualification $^{12}$.

12. De telles négociations pointent aussi vers la révision des contours de l'emploi convenable (FREYSSINET, 2000 ; LAVITRY, 2012), la réduction des exigences en matière de salaire, de métier, d'horaire, de contrat, étant soutenue par une discussion sur la valeur des qualités acquises, ou plus exactement sur les possibilités de les valoriser sur le marché du travail. 
D'après les résultats de l'enquête $O$ fer 2016, les recruteurs sont peu disposés à accorder de l'importance aux diplômes lors de l'accès aux emplois non qualifiés. En effet, à la question de savoir s'ils considèrent que le diplôme de la personne recrutée est ajusté à leur besoin, la moitié des recruteurs répondent qu'ils n'avaient pas d'attente à ce sujet lorsqu'il s'agit de pourvoir un emploi non qualifié. Cette indifférence se révèle particulièrement forte au moment du recrutement des nettoyeurs (69 \%). L. Tranchant remarque de son côté que le diplôme ne joue aucun rôle dans la sélection des intérimaires de la logistique.

F.X. Devetter et E. Puissant insistent pour leur part sur le rôle profondément ambivalent que jouent les diplômes dans les emplois à domicile, où leur valeur n'est pas pleinement reconnue. Et pourtant, la professionnalisation du secteur est soutenue par les pouvoirs publics qui encouragent aussi le recours à la validation des acquis de l'expérience (VAE). Mais les auteurs précisent que, pour augmenter leurs chances d'être recrutées, certaines diplômées ont tendance à s'en cacher, pour ne pas renchérir le coût de l'embauche. En effet, les diplômes restent facultatifs mais sont partiellement reconnus dans les conventions collectives des associations, tandis qu'ils n'ont aucune valeur établie dans les entreprises à but lucratif et dans les centres d'action sociale. Dans les procédures d'agrément des assistantes maternelles, on relève également que rien n'oblige les candidats à présenter des diplômes (VOZARI, 2014). Il n'empêche : les femmes diplômées, que leur diplôme soit en lien ou non avec l'activité, sont nettement plus souvent agréées que les autres, comme si leurs études offraient plus de garanties que les autres critères (taille du logement, respect des règles de sécurité ou d'hygiène). Et les ressources scolaires sont aussi valorisées au moment de l'embauche des aides à domiciles, sans pour autant être rétribuées.

\section{La recherche de qualités ajustées aux situations de travail}

Quelles attentes formulent les employeurs à l'égard de la main-d'œuvre recherchée ? L'absence de qualification des postes de travail pourrait laisser penser que ces attentes pointent vers des qualités génériques, non spécifiques. Pourtant, même les exigences les plus communes et répandues ne forment pas un tout homogène. La disponibilité, par exemple, est une qualité particulièrement recherchée pour faire face aux demandes des clients, que ce soit dans la logistique, l'hôtellerie-restauration, l'action sociale, la distribution ou le commerce. Elle est en phase avec l'urgence des recrutements, l'importance du turnover, le recours à des contrats courts et à l'intérim. Elle fait partie des questions prioritaires soumises aux candidats qui fréquentent les salons de recrutement dédiés à la main-d'œuvre peu ou pas qualifiée (MARCHAL et al., 2017). Mais ce que montrent les textes de ce dossier, c'est que la diversité des activités prises en charge réclame des qualités ajustées aux conditions d'exercice de ces activités. Le terme même de disponibilité recouvre des exigences différentes d'une activité à l'autre, et selon qu'il s'agit d'intérimaires ou de salariés, de temps partiels ou de saisonniers. 
La disponibilité temporelle est au cœur du texte de P. Grimaud, qui insiste sur le fait qu'elle ne donne pas lieu à une reconnaissance matérielle ou symbolique dans les emplois du commerce. Elle passe par l'acceptation d'horaires décalés et variables d'un jour à l'autre ou d'une semaine à l'autre, s'étirant le soir, occupant potentiellement les samedis, dimanches et jours fériés. Être disponible dans les emplois non qualifiés du secteur, c'est aussi accepter de ne pas avoir d'emprise sur son temps libre ${ }^{13}$. Il s'agit donc d'un critère déterminant au moment de l'embauche, conduisant à privilégier les étudiants pour travailler à temps partiel ou le week-end. Le « succès » des étudiants se retrouve dans d'autres secteurs pour les mêmes raisons : dans la grande distribution (RIEUCAU, SALOGNON, 2013) ou dans l'hôtellerie-restauration, secteur traité par S. Montchatre, tout particulièrement lorsqu'il s' agit d'emplois saisonniers ${ }^{14}$. Dans la logistique, L. Tranchant souligne lui aussi que la disponibilité est un critère premier pour évaluer l'employabilité des candidats qui se présentent dans les agences d'intérim. Mais il s'agit là d'une disponibilité immédiate, recouvrant à la fois la capacité à se libérer à l'instant même où une mission est proposée, y compris la nuit, et une capacité à se déplacer pour se rendre sur les lieux de travail d'affectation, ce qui suppose de posséder un véhicule. La disponibilité dont doivent faire preuve les aides à domicile recouvre encore une autre réalité, puisqu'il s'agit de se tenir prêt à répondre à des demandes très variables de la part des employeurs et d'accepter de faire un travail différent et ajusté à chaque foyer. L'exigence de disponibilité est structurelle dans les services relationnels, où elle prend des formes particulièrement contraignantes pour les salariés à temps partiel et où elle est accentuée par une exigence latente de dévouement difficilement répressible ou contrôlable (JANY-CATRICE, 2007).

À chaque métier correspondent aussi des qualités différentes qu'il faut pouvoir déceler au moment de l'embauche, comme le fameux sourire que l'on s'attend à voir sur les lèvres des hôtesses d'accueil (SCHÜTZ, 2018). S. Monchatre insiste sur l'importance des qualités physiques et morales dans le secteur de l'hôtellerie-restauration. L'apparence et la tenue vestimentaire, contrôlées sur une photo ou lors du dépôt de CV, semblent jouer un rôle majeur pour ces emplois exposés au regard de la clientèle ${ }^{15}$. La jeunesse offre des garanties de ce point de vue, parce qu'elle contient la promesse de posséder énergie et résistance. Du côté des qualités morales, c'est plutôt la fidélité qui est recherchée, afin de limiter les coûts du turnover. Et les trajectoires sont étudiées à l'aune de cette variable, en interrogeant les candidats sur de possibles instabilités et en cherchant à jauger leurs aptitudes à devenir des « obligés », pour reprendre les

\footnotetext{
13. La disponibilité produit ensuite des effets tout au long de la relation d'emploi pour évaluer les salariés dont les promotions sont conditionnées par leur capacité à accepter des horaires flexibles. Par ricochet, un défaut de disponibilité peut conduire à dévaloriser la main-d'œuvre permanente, désignée par son manque de flexibilité.

14. D'après Ofer 2016, au moment de leur recrutement, un bon tiers des personnes (en moyenne) sont en formation ou inactives. Cette proportion s'élève à $44 \%$ lors du recrutement des employés non qualifiés du commerce et à $55 \%$ dans l'hôtellerie-restauration.

15. Notons que les pratiques de sélection sont cohérentes avec les critères à déceler. L'apparence peut se contrôler de plusieurs manières et notamment au moment de l'entretien comme c'est le cas sur les postes d'accueil où elle joue un rôle déterminant (HIDRI NEYs, REMICHI, 2015).
} 
termes de l'auteure. Dans la logistique, la résistance physique importe beaucoup. Mais L. Tranchant montre que bien d'autres critères sont aussi pris en compte au moment de l'inscription de l'intérimaire. Le questionnement porte sur l'expérience, la détention du certificat d'aptitude à la conduite en sécurité (Caces : le permis cariste) ou la possession d'un véhicule. S'y ajoute une attention aiguisée à l'allure de celui qui passe la porte de l'agence. Ce repérage est censé permettre d'opérer un tri entre ceux qui sont motivés et ceux qui ne le sont pas, ceux qui paraissent «mollassons » ou prêts à accepter des pénibilités. La sélection ne s'arrête pas là pour autant. Elle peut s'accompagner de tests et se double systématiquement de la mise à l'épreuve de la disponibilité du candidat à l'occasion de chaque convocation, comme nous l'avons vu. Elle se prolonge ensuite durant chaque mission, où c'est alors la mesure de la productivité de l'intérimaire qui prend le relais. Et c'est à partir de cet étalon que se décident finalement son maintien et son rappel pour d'autres missions. Cette sélection en différentes étapes signale l'existence de recrutements routiniers et équipés, faisant intervenir des tests et des professionnels qui cherchent à gagner en efficacité. De telles caractéristiques se retrouvent dans le secteur des services à domicile où les employées subissent de nombreux contrôles préalables à leur embauche.

\section{Les conditions de la sélection}

L'attention portée à des qualités peu codifiées, souvent désignées sous les termes de « savoir-être » ou de «soft skills », est déterminante en l'absence de qualités formalisées. Dans le cas du travail non qualifié, la confrontation aux difficultés afférentes à leur détection a débouché sur le développement de modes de recrutement qui visent à détourner l'attention que les recruteurs portent aux CV écrits : MRS (méthode de recrutement par simulation), IOD (intervention sur l'offre et la demande), CV vidéo ou questionnaires (MARCHAL, 2015). Mais tous les recruteurs ne sont pas disposés à faire de tels investissements. En effet, les pratiques de recrutement varient grandement avec les types d'entreprises qui recrutent, en termes de taille et de secteur et avec les moyens consacrés à cette activité (MARCHAL, RIEUCAU, 2010). Elles varient également avec le niveau de qualification du personnel à recruter, les employeurs étant davantage disposés à investir pour recruter des cadres ou pourvoir des emplois à durée indéterminée. Pourtant, les modes de sélection sont loin d'être uniformes lorsqu'il s'agit de pourvoir des emplois non qualifiés.

Les résultats de l'enquête Ofer 2016 laissent apparaitre un premier mode de sélection, particulier aux emplois de l'aide à domicile : il consiste à ouvrir en amont toutes les possibilités d'attirer une main-d'œuvre abondante, pour opérer une sélection drastique en aval. Nous avons vu en effet que les prestataires de services à domicile constituent des viviers de candidatures, alimentés par toutes sortes de canaux sollicités en parallèle, pour faire face à des demandes imprévues. Les candidates ${ }^{16}$ potentielles

16. Nous utilisons le genre féminin pluriel pour ces emplois qui ont donné lieu au recrutement de femmes dans $96 \%$ des cas. 
sont donc nombreuses, mais moins souvent introduites par des réseaux qu' ailleurs, ce qui nécessite de contrôler rigoureusement leurs qualités et leurs compétences. Comme l'indique le graphique 2, rien ne semble laissé de côté, puisque leur sont très souvent demandées, outre un $\mathrm{CV}$ et une lettre de motivation, des références dans des proportions supérieures aux autres emplois non qualifiés et en comparaison avec ce qui est demandé en moyenne à l'ensemble des candidats ${ }^{17}$. Ces résultats sont confirmés par une analyse « toutes choses égales par ailleurs ${ }^{18}$ » : on demande significativement plus fréquemment aux personnes recrutées pour un emploi dans les services à domicile (que pour un autre emploi non qualifié), de fournir des diplômes, des permis de conduire et des certificats (titre de séjour, pièce d'identité, carte vitale, certificat médical).

En plus de ces éléments à fournir, les personnes recrutées dans les services à domicile ont, de façon significative, passé bien plus de tests de connaissance ou d'intelligence et de tests de lecture, d'écriture ou de calcul que les autres ${ }^{19}$. L'objectif est de s'assurer qu'elles sauront se servir des produits et appareils ménagers ou lire les modes d'emploi et qu'elles auront les réactions adaptées dans d'éventuelles situations d'urgence. Il faut également contrôler leur capacité à respecter des consignes de sécurité et d'hygiène. Mais ce n'est pas tout : des tests de situations de travail sont également organisés dans $64 \%$ des recrutements des employées des services à domicile, afin de mesurer leur productivité attendue dans les tâches ménagères. Ainsi, la sélection à l'entrée sur ce type d'emplois est particulièrement consistante.

Dans d'autres types d'emplois non qualifiés, elle n'est pas moins forte, mais elle s'effectue différemment. Un autre mode de sélection consiste à miser sur les réseaux de relation. Rappelons à ce sujet que le rôle des réseaux diffère fortement selon les secteurs et qu'il est très prononcé dans la recherche d'ouvriers non qualifiés (dans le nettoyage et le bâtiment). Les réseaux contribuent à réguler les flux de candidatures et à présélectionner la main-d'œuvre, ce qui allège le coût de la sélection des postulants par la suite. Il s'agit souvent de réseaux de personnes immigrées, mobilisées par le personnel en place, ce qui offre des garanties sur les qualités des futurs embauchés. Ces mécanismes entretiennent des ségrégations à caractère ethnique, tout en étant pourvoyeurs d'une main-d'œuvre souvent mobile et difficile à contacter pour les employeurs, même s'ils ramènent aussi vers les recruteurs des anciens stagiaires ou intérimaires ou travailleurs saisonniers. D'après l'enquête Ofer 2016, la proportion de nouvelles recrues déjà connues par l'employeur s'élève à $35 \%$ pour l'ensemble des recrutements, ce qui n'est pas négligeable. Elle est encore plus importante dans le bâtiment où la moitié des ouvriers non qualifiés ne sont pas des inconnus pour l'entreprise qui les recrute. Pour les nettoyeurs aussi cette proportion est forte (42\%) tandis que, a contrario, elle est particulièrement basse (18\%) pour les aides à domicile.

\footnotetext{
17. Maxime BERGEAT et Véronique RÉmy (2017) présentent de façon exhaustive les éléments demandés à l'ensemble des candidats et les méthodes utilisées pour les sélectionner, d'après Ofer 2016.

18. Cette analyse tient compte des principales caractéristiques des candidats (sexe, âge, diplôme, origine, etc.) et des emplois (temps de travail, salaire et type de contrat).

19. Voir note 6.
} 
GRAPHIQUE 2 - Éléments demandés à l'occasion du recrutement pour les cinq types d'emplois non qualifiés

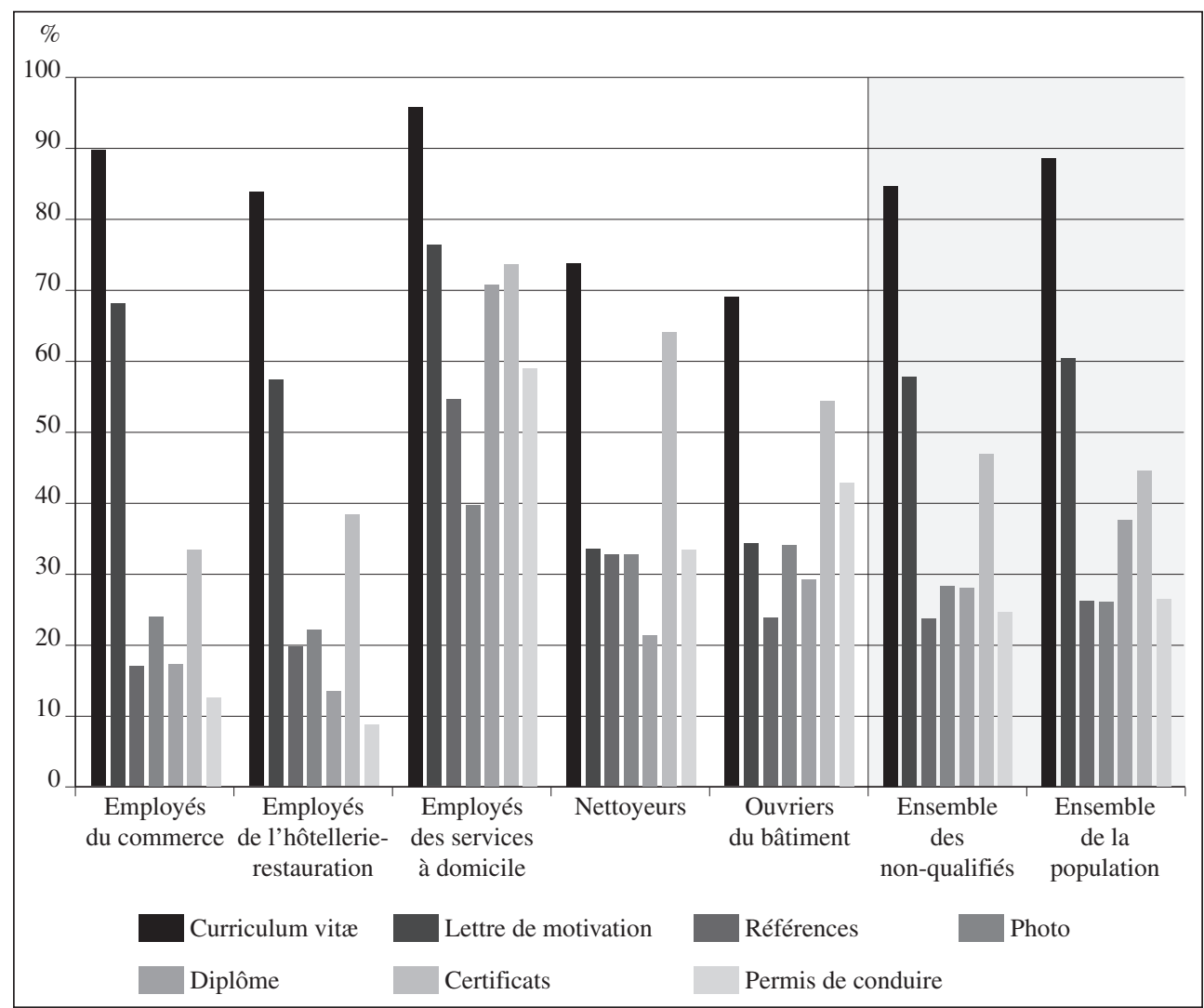

Lecture : dans $90 \%$ des recrutements des employés du commerce non qualifiés, il est demandé de fournir un CV.

Champ : ensemble des nouveaux recrutements en CDI ou CDD de plus d'un mois dans les établissements d'au moins un salarié du secteur concurrentiel ; France entière. Données pondérées.

Source : Ofer, Dares, 2016.

Un autre mode de sélection plus ou moins typique de certains emplois non qualifiés peut être repéré. Il consiste à mettre directement à l'essai les nouvelles recrues sur des postes de travail. De telles pratiques s'observent plus souvent dans les petites structures où le recruteur se trouve seul pour prendre des décisions. Ainsi, les mises à l'essai sont significativement plus fréquentes dans l'hôtellerie-restauration, pour confirmer la sélection. Dans le bâtiment aussi, les mises à l'essai se pratiquent pour contrôler l'attitude au travail ou la productivité des ouvriers, manœuvres ou manutentionnaires (33\% des cas), alors que c'est moins courant dans le commerce (23\%). Les mises à l'essai sont une manière d'opérer une sélection de la main-d'œuvre sur le tas. On peut supposer qu'elles sont également une source d'auto-sélection dans les emplois qui nécessitent peu ou pas d'apprentissage, en mettant le candidat en situation d'éprouver lui-même sa capacité à résister à la pression ambiante, dans la restauration rapide par exemple, ou à supporter la dureté physique des conditions de travail. 


\section{La fragilité des travailleurs les moins qualifiés dans la division du travail}

Nous avons vu que la non-qualification est le produit d'opérations multiples qui se déploient largement à distance et en amont des situations et des lieux de travail. Pourtant, ceux-ci sont aussi des espaces de qualification, où tend à s'actualiser et à se renforcer la non-reconnaissance, même si, là encore, une certaine variabilité existe. Les travailleurs occupant des postes non qualifiés ont rarement des positions centrales dans les organisations productives, ils sont au contraire repoussés en périphérie. Cette mise aux marges accentue leur non-qualification et cela, d'autant plus que leurs capacités de résistance collective, et plus largement d'action organisée, sont réduites. Nous soulignons d'abord comment les divisions statutaires participent à la mise aux marges des travailleurs non qualifiés, puis montrons que leur fragilité est renforcée par de multiples jeux sur les limites entre qualification et non-qualification, développés au coeur de la division du travail.

\section{Divisions statutaires et mises à l'écart}

Partant du cas des aides à domicile, dont la diversité des trajectoires est finement analysée (AVRIL, 2014), Olivier SCHWARTZ (2014) remarque combien cette diversité fait obstacle à leur mobilisation : «l'occupation d'un emploi du bas de l'échelle ne garantit jamais, et garantit moins que jamais aujourd'hui, que ceux qui l'occupent vont se constituer en communauté soudée » (SCHWARTZ, 2014, p. 283). On sait aussi que la participation syndicale ou politique est faible chez des travailleurs qui ne nourrissent pas de sentiment de leurs propres intérêts et a fortiori de sentiment d'appartenance de classe. Si celle-ci est « objectivement consistante, elle apparaît subjectivement éclatée » (AMOSSÉ, CHARDON, 2006, p. 223). En pénétrant au plus près des situations de travail, ce dossier permet d'identifier des mécanismes producteurs de cet éclatement et de cette faible cohésion, en insistant sur des caractéristiques des modes de gestion des travailleurs non qualifiés qui entravent l'émergence de collectifs. Il en résulte des difficultés pour ces travailleurs à conquérir des qualités et à se mobiliser pour leur reconnaissance, mais cela produit aussi une différenciation des attitudes à l'égard du travail et face à l'enjeu de la conquête de qualités.

La faiblesse des conditions favorables à l'intégration dans des collectifs de travail résulte de multiples facteurs, relevant tout autant des statuts d'emploi (avec la flexibilité et le turnover comme nous l'avons déjà noté) que des conditions de travail. Souvent ces deux éléments se combinent et se renforcent, comme l'illustre le cas des aides à domicile étudié par F.-X. Devetter et E. Puissant. Les analyses qu'ils esquissent des situations de travail montrent un cumul de facteurs contribuant à maintenir le travail à distance de la qualification, et les travailleurs à distance de la mobilisation pour la qualification : ceux-ci sont dispersés géographiquement et largement isolés dès lors qu'ils travaillent au domicile de particuliers ; ils accomplissent une activité perçue 
comme proche de tâches domestiques traditionnellement non rétribuées et donc sans guère de valeur ; ils sont souvent embauchés sous le régime de l'emploi direct dans lequel seules comptent dans le calcul de la rémunération les interventions à domicile, à l'exclusion de toute autre composante du temps de travail ; ils effectuent un travail qui touche à l'intimité des corps et réclame de la discrétion de sorte qu'il est difficile de mettre en valeur ce dont il est convenu de ne pas parler. Dans ces conditions, il apparaît difficile de prendre une part active dans des processus de qualification, tant à l'échelle de la professionnalisation et de la formalisation des savoirs en référentiels ou en diplômes (DEMAZIÈre, 2009) qu'à l'échelle de négociations collectives au niveau des branches ou des entreprises prestataires.

Les divisions statutaires contribuent de la même manière à entraver la cohésion des collectifs de travail, d'autant plus quand elles sont actualisées au cœur des situations de travail, comme le montre l'évocation par F.-X. Devetter et E. Puissant du cas des agents d'entretien dans les collèges, où la coexistence d'agents publics et d'agents de sociétés prestataires est réglée par la prescription de cadences de travail spécifiques et par la différenciation catégorielle des salles de pause. Les journaliers de Chicago sont eux aussi considérés comme des « salariés périphériques » qui ne disposent ni des mêmes droits que les autres, ni des mêmes protections, ni des mêmes possibilités de circuler dans les locaux des entreprises où ils travaillent (CHAuvin, 2010). Le poids des statuts d'emploi dans la régulation des relations de travail quotidiennes est bien mis en évidence dans le cas d'entreprises de logistique qui font un appel massif à l'intérim. Le texte de L. Tranchant montre qu'alors, l'intérim n'est pas constitué comme une antichambre du contrat (FAURE-GUICHARD, FourniER, 2001) mais qu'il instaure une catégorie spécifique de travailleurs. Cette spécificité excède de beaucoup le statut, puisque les intérimaires subissent une surintensité du travail et sont affectés aux tâches les plus pénibles, les plus dévalorisées, les plus difficiles au regard des objectifs de productivité à atteindre. Ils occupent des postes non qualifiés, dont les propriétés sont encore dégradées dans le procès de division du travail. Cette gestion différenciée des prescriptions n'épargne pas les permanents, puisque ceux-ci n'ont pas un traitement uniforme, les meilleures tâches étant affectées prioritairement à ceux qui sont considérés comme les plus loyaux, selon un mécanisme répandu de rétribution différentielle, matérielle ou symbolique, des engagements au travail (BIGI et al., 2015).

\section{Les jeux sur les limites de la (non-)qualification}

Les mécanismes de division du travail et des travailleurs traversent la nébuleuse de la non-qualification, et ne se limitent pas à tracer la frontière entre qualifiés et non qualifiés. Ils peuvent d'ailleurs être observés dans des secteurs relativement indifférenciés du point de vue de la qualification des postes de travail, comme celui du commerce où la distinction entre employés qualifiés et non qualifiés n'est pas opérante. Partant de ce constat, P. Grimaud met en évidence dans son texte des mécanismes de différenciation dans un monde du travail tellement plat que les écarts de salaires entre employés 
sont limités. Son étude montre comment l'extension du travail dominical introduit de nouvelles divisions parmi les employés, et redéfinit les termes dans lesquels se pose la qualification du travail. La gestion du développement du travail dominical s'organise dans deux directions : le recrutement d'employés pour le week-end qui forment des « équipes d'appoint » distinctes des employés classiques, et le recours à ces derniers sur la base du volontariat. Les conséquences sur la qualification sont multiples. Chez les employés classiques et leurs représentants syndicaux, émerge un sentiment de déqualification et de dégradation du métier, nourri par le fait que les employés d'appoint ont des caractéristiques sociales spécifiques (étudiants, jeunes sans diplôme, seniors, etc.) qui sont généralement associées aux « petits boulots » occasionnels et à un travail de faible valeur (BÉDUwÉ, GIRET, 2004 ; PINTO, 2014). L'émergence d'autres catégories de main-d'œuvre, qui ont un autre rapport au travail, plus alimentaire et instrumental, participe ainsi à la dévalorisation du travail. Par ailleurs, les employés classiques sont désormais distribués en deux catégories : ceux qui ont accepté le travail dominical et les autres. S'ils accomplissent des tâches comparables, ils se différencient sur le plan salarial, compte tenu des majorations sensibles associées au travail dominical et du caractère plat des carrières salariales : dès lors, le travail du dimanche place de facto en haut de la pyramide des salaires, ce qui en fait un nouveau principe de qualification salariale. L'extension du travail dominical introduit ainsi de nouvelles divisions et dévalorisations : dévalorisation des permanents du fait du recrutement de salariés atypiques ordinairement affectés à des emplois sans qualification, dévalorisation des permanents ne travaillant pas le dimanche au regard de ceux qui le font. Cela renforce la division et la concurrence, en faisant émerger, dans un monde du travail relativement indifférencié et valorisant les savoir-faire de vendeurs spécialisés, des hiérarchies fondées sur le salaire et la flexibilité, annonciatrices d'une potentielle redéfinition de la qualification et de la non-qualification.

Le développement de la polyvalence est un autre moyen de déplacer les coordonnées de la qualification du travail, en introduisant des jeux multiples entre contenu des tâches et désignation des postes. Dans certains cas, cela conduit à une interchangeabilité des travailleurs, qui annihile toute perspective de qualification. Ainsi, dans les supermarchés alimentaires low cost, la « succession (des activités) est toujours incertaine et changeante » (GARDES, 2018, p. 5). La polyvalence y est poussée à un degré élevé d'intensité. Les salariés circulent d'une activité à l'autre au cours de la journée de manière à éviter tout temps mort, et se voient attribuer un secteur (produits frais, secs ou entretien) chaque matin, en sorte qu'ils prennent en charge tous les rayons à tour de rôle. Ils commencent leur journée par des tâches de manutention en s'interrompant selon un ordre déterminé lorsque des clients passent en caisse, et ils contribuent non seulement au travail de mise en rayon mais aussi au nettoyage des sols, tout en restant constamment disponibles aux demandes de la clientèle. Dans une telle organisation du travail, la notion même de poste disparaît, les salariés partagent les pénibilités par roulement et peuvent être facilement comparés et remplacés au regard de leur niveau de productivité. Plus, les hiérarchies entre employés sont aplaties, 
ce qui réduit considérablement les potentialités de carrière puisque la seule option promotionnelle possible est le passage dans l'encadrement.

Les jeux sur la division du travail sont infinis. Ainsi, dans son texte, $\mathbf{S}$. Monchatre décrit comment dans certains segments du secteur de l'hôtellerie-restauration, certaines tâches sont redistribuées afin de faire face à des pénuries potentielles de maind'œuvre : par exemple la plonge (ou le nettoyage), qui est une activité dévalorisée et souvent dévolue à une main-d'œuvre infériorisée et sans qualité, peut être affectée à des employés qualifiés tels les cuisiniers afin de pallier des difficultés de recrutement. L'introduction de la polyvalence répond à des enjeux différents selon les contextes de travail, mais elle n'est pas sans implication sur le processus de qualification. Dans la logistique étudiée par $\mathbf{L}$. Tranchant, ces jeux se déploient sur un terrain balisé par deux couples : l'un relatif aux statuts et à la qualification des personnes différencie permanents et intérimaires, l'autre relatif aux postes et à la qualification du travail différencie caristes et préparateurs de commande. Les deux premiers termes (permanents et caristes) sont qualifiés au regard des seconds (intérimaires et préparateurs) qui ne le sont pas, mais la division du travail est plus complexe que cette partition. Des intérimaires missionnés comme caristes effectuent aussi des tâches de préparateur, ce qui représente une déqualification (dans le rapport entre le poste, mais aussi la personne car pour être recruté comme cariste, il faut détenir le permis correspondant, et les tâches). Inversement, des permanents recrutés comme préparateurs mais ayant obtenu le permis cariste peuvent être partiellement affectés à un poste de cariste, ce qui représente une autre forme de non-qualification (dans le décalage, inversé cette fois, entre le poste, qui nominalement ne change pas, et les tâches). Cette dernière pratique contribue aussi à attiser la concurrence entre préparateurs permanents pour accéder à des tâches de cariste et éventuellement changer de poste. Ces multiples déplacements montrent combien les salariés ont peu de prise sur le processus de reconnaissance des qualifications : ils sont réduits, à partir de positions différentes, à subir des jeux qui entretiennent et renforcent des formes, multiples, de non-qualification.

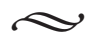

Les contributions réunies dans ce dossier soulignent que la fabrication du travail non qualifié se joue à plusieurs niveaux. Elle se décline au plus près des situations de travail où les qualités des travailleurs sont mises à l'épreuve à travers la gestion des tâches et des postes ou la recomposition de la division du travail. Mais elle s'observe aussi en amont, dans les opérations de recrutement et d'affectation au cours desquelles les qualités des candidats sont scrutées, évaluées, négligées, minorées et, ce faisant, produites à travers leur prise en compte ou leur ignorance. Elle se décline enfin à une échelle plus large, sur les marchés du travail, là où les entreprises en quête de main-d'œuvre ainsi que les intermédiaires publics et privés de l'emploi développent des pratiques et mobilisent des outils multiples pour configurer les qualités des actifs cherchant un poste. 
Prendre appui sur la sociologie du travail, des professions et des marchés permet de saisir la complexité de cette dynamique de la qualification, qui se déploie à l'intérieur des organisations et sur les lieux de travail, comme aux portes des entreprises et à travers les transactions marchandes.

Pour prolonger ce dossier, il faudrait systématiser l'exploration de ces différents niveaux et analyser comment ils s'articulent selon les métiers ou les secteurs. Cela permettrait de saisir toute l'amplitude de la fabrication du travail non qualifié et la variété des mécanismes qui contribuent à son développement actuellement. Ainsi, dans les cas où les éléments propices à la dévalorisation des compétences se retrouvent à tous les niveaux, les processus de déqualification à l'œuvre sont particulièrement puissants et difficiles à enrayer. Cet aspect a été bien étudié à propos de l'aide à domicile qui rencontre de lourds obstacles à sa professionnalisation. Mais l'on aurait tort d'en tirer des enseignements généraux car de tels obstacles ne se retrouvent pas réunis dans d'autres activités. La comparaison des conditions de recrutement des cinq populations étudiées (employés du commerce, de l'hôtellerie-restauration, des services à domicile, nettoyeurs et ouvriers du bâtiment), toutes non qualifiées, laisse apparaître de fortes variations et des marges de manœuvre différentes d'une population à l'autre. La fabrication du travail non qualifié est donc multiforme et variable selon les secteurs. Mais de manière transversale, il importe à nos yeux d'en rendre compte comme du résultat, jamais entièrement stabilisé, de l'activité des employeurs et des salariés, de leurs représentants respectifs, des intermédiaires, des recruteurs, autant d'acteurs qui sont engagés dans les mondes du travail et contribuent à leurs régulations. Une telle perspective permet de ne pas rabattre la qualification et la non-qualification ni sur des qualités acquises et accumulées lors des parcours individuels, ni sur des qualités requises et attachées à des postes de travail, mais de les concevoir théoriquement et de les saisir empiriquement comme des qualités conquises et valorisées à travers les rapports sociaux à l'œuvre sur les marchés du travail. Elle permet aussi de comprendre pourquoi, dans la période actuelle, la qualification se trouve fragilisée.

\section{BIBLIOGRAPHIE}

Аввотт A. (1988), The System of Professions. An Essay on the Division of Expert Labor, Chicago, University of Chicago Press.

Amossé T., ChARdon O. (2006), « Les travailleurs non qualifiés : une nouvelle classe sociale ? », Économie et statistique, $\mathrm{n}^{\circ}$ 393-394, pp. 203-229.

Amossé T., Perraudin C., Petit H. (2011), « Mobilité et segmentation du marché du travail : quel parcours professionnel après avoir perdu ou quitté son emploi ? », Économie et statistique, $\mathrm{n}^{\mathrm{o}} 450$, pp. 79-105.

Avril C. (2014), Les Aides à domicile. Un autre monde populaire, Paris, La Dispute.

Barron P., Bory A., Chauvin S., Jounin N., Tourette L. (2011), On bosse ici. On reste ici ! La grève des sans-papiers : une aventure inédite, Paris, La Découverte. 
BÉDuwÉ C., GiRET J.-F. (2004), « Le travail en cours d'études a-t-il une valeur professionnelle ?», Économie et statistique, $\mathrm{n}^{\mathrm{o}}$ 378-379, pp. 55-83.

Béraud M., Eydoux A., Fériel É., Higelé J.-P. (2015), « L’instrumentalisation du RSA par les employeurs. Un débat révélateur d'un malentendu sur l'emploi non qualifié », Les Notes de l'Institut européen du salariat, $\mathrm{n}^{\circ} 37$.

BergeAt M., RÉMY V. (2017), « Comment les employeurs recrutent-ils leurs salariés ? », Dares analyses, $\mathrm{n}^{\mathrm{O}} 064$.

Bergeat M., Fondeur Y., Minni C., RÉMY V. (2018), « Mobiliser Internet pour recruter : quelles sont les pratiques des employeurs ? », Dares analyses, $\mathrm{n}^{\circ} 032$.

Bigi M., Cousin B., Méda D., Sibaud L., Wieviorka M. (2015), Travailler au XXI siècle : des salariés en quête de reconnaissance, Paris, Robert Laffont.

Burnod G., Chenu A. (2001), « Employés qualifiés et non qualifiés : une proposition d'aménagement de la nomenclature des catégories socioprofessionnelles », Travail et Emploi, $\mathrm{n}^{\circ} 86$, pp. 87-105.

CHAPPERT F., Therry P. (2017), Photographie statistique des accidents de travail, des accidents de trajets et des maladies professionnelles en France selon le sexe, entre 2001 et 2015. Des tendances d'évolution différenciées pour les femmes et pour les hommes, Lyon, Anact.

Chardon O. (2001), « Les transformations de l'emploi non qualifié depuis vingt ans », Insee première, $\mathrm{n}^{\mathrm{o}} 796$.

Chauvin S. (2010), Les Agences de la précarité. Journaliers à Chicago, Paris, Seuil.

Cresson G., Gadrey N. (2004), «Entre famille et métier : le travail du care », Nouvelles Questions féministes, vol. 23, n 3, pp. 26-41.

DÉCosse F. (2012), « Maraîchage : "Non aux contrats bidon !” », Plein droit, nº 94, pp. 36-40.

DEMAZIÈRE D. (2009), « Professionnalisations problématiques et problématiques de la professionnalisation », Formation emploi, $\mathrm{n}^{\circ} 108$, pp. 89-99.

Demazière D., GadÉA C. (dir.) (2009), Sociologie des groupes professionnels. Acquis récents et nouveaux défis, Paris, La Découverte.

Desrosières A., ThéVEnot L. (1988), Les Catégories socioprofessionnelles, Paris, La Découverte.

Devetter F.-X., Jany-Catrice F., Ribault T. (2009), Les Services à la personne, Paris, La Découverte.

Dussuet A. (2016), « Genre, frontières du travail domestique et marges du salariat. Le cas des aides à domicile », Revue française de socio-économie, $\mathrm{n}^{\circ}$ 17, pp. 123-141.

Estrade M.-A. (2008), « Une prospective socio-économique du travail et de l'emploi peu qualifié », in Insee, L'Emploi, nouveaux enjeux, édition 2008, pp. 27-39.

FAure-Guichard C., Fournier P. (2001), « L'intérim, creuset de main-d'œuvre permanente ? », Genèses, n $^{\circ} 42$, pp. 26-46.

FlAMAND J. (2016), «Les transitions professionnelles, révélatrices d'un marché du travail à deux vitesses », La Note d'analyse, n ${ }^{\circ} 50$, France stratégie. 
FreysSinEt J. (2000), « Plein emploi, droit au travail, emploi convenable », Revue de l'Ires, $\mathrm{n}^{\mathrm{o}} 34$, pp. 27-58.

GARDES C. (2018), « Le coût des prix bas. Travailler dans le hard discount alimentaire », La Nouvelle Revue du travail [en ligne], $\mathrm{n}^{\circ} 12$ : https://journals.openedition.org/nrt/3515, consulté le 25 janvier 2019.

Guichard-Claudic Y., Kergoat D., Vilbrod A. (dir.) (2008), L'Inversion du genre. Quand les métiers masculins se conjuguent au féminin... et réciproquement, Rennes, Presses universitaires de Rennes.

Hidri Neys O., Remichi Y. (2015), «"Privé versus Public”. Le poids des apparences dans le recrutement du personnel d'accueil », La Nouvelle Revue du travail [en ligne], $\mathrm{n}^{\circ} 7$ : https://journals.openedition.org/nrt/2398, consulté le 28 janvier 2019.

JANY-CATRICE F. (2007), « La dévalorisation des services "relationnels" dans les pratiques et les conventions dominantes », L'Homme et la Société, n ${ }^{\circ}$ 163-164, pp. 15-34.

Jounin N. (2004), « L'ethnicisation en chantiers. Reconstructions des statuts par l'ethnique en milieu de travail », Revue européenne des migrations internationales, vol. 20, nº 3, pp. 103-126.

KeRgOAT D. (2010), «Le rapport social de sexe. De la reproduction des rapports sociaux à leur subversion », in Bidet-Mordrel A. (coord.), Les Rapports sociaux de sexe, Paris, Presses universitaires de France, pp. 60-75.

LAVITRY L. (2012), « Le jugement d'employabilité : un nouveau savoir pour gérer les chômeurs ?», Sociologies pratiques, $\mathrm{n}^{\circ} 24$, pp. 53-65.

Le Feuvre N., Benelli N., Rey S. (2012), « Relationnels les métiers de service ? », Nouvelles Questions féministes, vol. 31, $\mathrm{n}^{\mathrm{O}} 2$, pp. 4-12.

Marchal E. (2015), Les Embarras des recruteurs. Enquête sur le marché du travail, Paris, Éditions de l'EHESS.

Marchal E., Remillon D., Rieucau G. (2017), « Éprouver le marché du travail dans les salons de l'emploi », Socio-économie du travail, n 2, pp. 101-129.

Marchal E., Rieucau G. (2010), Le Recrutement, Paris, La Découverte.

MARX K. ([1867] 1977), Le Capital. Livre premier, traduit par J. Roy, Paris, Éditions sociales.

Matus M., Prokovas N. (2014), « Du chômage à l'emploi : une mobilité professionnelle importante et complexe », Éclairages et synthèses, n ${ }^{\circ}$ 02, Pôle emploi.

MÉda D., Vennat F. (2004), Le Travail non qualifié. Permanences et paradoxes, Paris, La Découverte.

Morice A., Ротот S. (dir.) (2010), De l'ouvrier immigré au travailleur sans papiers. Les étrangers dans la modernisation du salariat, Paris, Khartala.

PICART C. (2014), « Une rotation de la main-d'œuvre presque quintuplée en 30 ans : plus qu'un essor des formes particulières d'emploi, un profond changement de leur usage », Document de travail, $\mathrm{n}^{\circ} \mathrm{F} 1402$, Insee. 
PinTo V. (2014), À l'école du salariat. Les étudiants et leurs «petits boulots », Paris, Presses universitaires de France.

RÉMY V. (2017), « Pourquoi les employeurs choisissent-ils d'embaucher en CDD plutôt qu'en CDI ? », Dares analyses, $\mathrm{n}^{\circ} 070$.

REYSSAT F. (2016), « Quand espace et objet de travail se confondent. Le cas du nettoyage », $L a$ Nouvelle Revue du travail [en ligne], ${ }^{\circ} 9$ : https://journals.openedition.org/nrt/2888, consulté le 28 janvier 2019.

RieuCAU G., SALOGNON M. (2013), « Le recrutement dans la grande distribution : des pratiques ajustées ? », Revue de l'Ires, n 76, pp. 45-69.

Rose J. (2012), Qu'est-ce que le travail non qualifié ?, Paris, La Dispute.

Rosini P. (2012), «L'appel du travail. Technologies et mobilisation subjective d'intérimaires "non qualifiés" ", La Nouvelle Revue du travail [en ligne], $\mathrm{n}^{\circ} 1$ : https://journals.openedition. org/nrt/228, consulté le 28 janvier 2019.

Schüтz G. (2018), Jeunes, jolies et sous-traitées : les hôtesses d'accueil, Paris, La Dispute.

Schwartz O. (2014), « Postface», in Avril C., Les Aides à domiciles. Un autre monde populaire, Paris, La Dispute, pp. 267-284.

SCRINZI F. (2013), Genre, migrations et emplois domestiques en France et en Italie. Construction de la non-qualification et de l'altérité ethnique, Paris, Éditions Petra.

VOZARI A.-S. (2014), « Recruter de “bonnes” assistantes maternelles. La sélection à l'entrée d'un emploi féminin non qualifié », Sociétés contemporaines, $\mathrm{n}^{\circ}$ 95, pp. 29-54.

ZunE M. (2014), «Éditorial. De quoi les pénuries de main-d'œuvre sont-elles le nom ? », Revue française de socio-économie, $\mathrm{n}^{\mathrm{o}} 14$, pp. 5-14. 
